\title{
ELECTROENCEPHALOGRAPHIC CHANGES IN CEREBELLAR DEGENERATIVE LESIONS
}

\author{
BY
}

\author{
L. A. LIVERSEDGE and V. EMERY \\ From the Departments of Neurology and Encephalography, University of Manchester, \\ Manchester Royal Infirmary
}

Following the rapid extension of electroencephalography in the investigation of all forms of intracranial affection in clinical neurology, it soon became apparent that tumours in the posterior fossa greatly complicated the electroencephalogram. Numerous early publications, including the work of Grey Walter (1936, 1937, and 1938) and that of Bickford and Baldes (1947), gave conflicting information and seemed only to indicate that there was no pattern of electrical abnormality characteristic of a posterior fossa disturbance. However, Bagchi, Lam, Kooi, and Bassett (1952) reported their electroencephalographic experiences over a period of seven years gained from examining 37 patients, all of whom had proven posterior fossa tumours. Their findings indicated that 32 of these patients had abnormal records and that the abnormality consisted either of background slowing or of burst activity of one type or another, or else a combination of the two. Bagchi and his associates were not able to say what clinical or pathological factors were definitely responsible for these E.E.G. abnormalities. They thought that in any particular case neither the size nor the character of the tumour could be related either to the degree of slowing or to the prominence of the burst activity. Daly, Whelan, Bickford, and McCarthy (1953) found that the principal electrical abnormality seen in their series (comprising 66 patients with tumours of the posterior fossa, 12 who had tumours of the third ventricle, and a further nine who had obstructive hydrocephalus), consisted of rhythmic bilateral synchronous slow waves, though less often irregular arrhythmic waves might be seen. In this series they could find no correlation between the electroencephalographic abnormalities and the site of the lesion. Daly suggested, therefore, that hypertension in the third ventricle was of importance in the production of these abnormal rhythms. Dow (1956), reviewing this problem, concurred with this notion of third ventricular hypertension, but, as Bagchi et al. (1952) had pointed out, there are a number of ways in which the expansion of an infra- tentorial lesion may produce a change in the $\stackrel{\mathbb{D}}{\circ}$ electroencephalogram. In the first instance the postero-inferior portions of the cerebrum may be $\vec{\circ}$ subjected to direct contiguous pressure from the cerebellar tumour. Secondly, progressive obstruction $\vec{~}$ to the outflow of cerebrospinal fluid may produce? the third ventricular hypertension to which allusion? has been made. Finally, however, we must not for-iv get that involvement of the cerebello-cerebral pathways may influence remote cerebral electricalic activity and so produce a disturbance of the normal electroencephalographic patterns. The anatomicilo and physiological relationships between cerebrifi and cerebellar activity have been clearly demon- $z$ strated by the work of Smyth (1941), Snider (195\%, and Snider and Eldred (1952), and leads to the $\frac{\Phi}{3}$ suggestion that there will be some electrical interplay between the various connexions. Apart from the information available in relation to posterior fossa $\vec{\varnothing}$ tumours, there is little or no evidence of the way in:which other pathological processes in the posterior fossa may influence the E.E.G., although Moruzzi \& and Magoun (1949) have indicated that the reticular formation may well have a large part to play in the production of normal cerebral electrical activity, and, $\mathbb{\odot}$ therefore, of abnormal cerebral electrical patterns. Yet Russell Brain and Strauss(1955) include amongst disorders in which no electroencephalographic abnormality is noted, the degenerations of the cerebellum and of the spino-cerebellar pathways. For this reason it was thought that it would be profitable to collect what information might be available on? the subject of E.E.G. recordings in relation to 3 cerebellar and spino-cerebellar disturbances which are not primarily due to tumours. We have been 을 able to collect evidence of this nature in 21 patients on whom electroencephalography was carried out.

\section{Clinical Material}

Greenfield (1954) pointed out that it is not always possible, indeed it is rarely possible, to establish with certainty in life the particular type of cerebellar 
disorder to which a given case belongs. In his monograph, however, he divided the spino-cerebellar disorders into three main groups, namely, (1) predominantly spinal forms, (2) spino-cerebellar forms, and (3) predominantly cerebellar forms.

We have found it possible and convenient to group the 21 patients seen in this study under these three broad headings: two of the 21 belong to the spinal group, four to the spino-cerebellar, and the remaining 15 to the predominantly cerebellar types (Table I).

It was very interesting to note that in the spinal types the electroencephalographic recordings were within normal limits, the only notable feature being that random theta activity was present. In three of the four spino-cerebellar patients again the only abnormality noted was the tendency for theta outbursts to be present. The fourth of these (Case 6), who exhibited a pronounced cerebellar defect and also optic atrophy, in addition to the spinal manifestations, later developed epileptic attacks, both generalized and myoclonic in type. The E.E.G. in this case contained a good deal of high-voltage delta activity, situated in the posterior temporal and parieto-occipital areas on both sides. The interest of these findings hinges largely on the fact that the degenerative lesions of spinal distribution only do not appear to produce any form of electrical abnormality, and only cases with added cerebellar features develop abnormal electrical patterns. It is, therefore, all the more significant that one finds in group 3 (the predominantly cerebellar group) very definite E.E.G. disturbances. Table I summarizes the principal clinical findings in these cases, which include, in addition to the cerebellar degenerative disorders, one case of hyperpyrexial cerebellar degeneration, one case of left-sided cerebellar infarct, and three patients in whom myoclonic manifestations were present in addition to the obvious cerebellar defect. In three other cases the clinical evolution showed that, at a later stage, there was an extension of the disorder to extrapyramidal pathways other than the cerebellar connexions. The possible significance of this will be discussed later.

Analysis of the Cerebellar Group.-We discovered that in 13 of the 15 cases in this group, there was evidence of electroencephalographic abnormalities, which may be summarized as follows:-

(1) A tendency for slowing of the dominant rhythm in 12 of the 15 , with forward spread of this rhythm in 11 of these; (2) an excess of theta activity in all 15 of the cases; (3) delta activity was noted in 12 cases, and in four of them it was at times focal. In six cases the delta activity was bilaterally synchronous, its situation being in two cases posterior, in two anterior, and in two central. In the three other cases of the group some delta activity was noted, but it was not very prominent. It is to be noted that the theta and delta activity could be inhibited by visual stimuli in four of the 15 cases. We were particularly interested to observe that in Case 9, in which there was an infarct in the left cerebellar lobe, these abnormal features were very prominent, for the dominant rhythm was between 4 and $7 \mathrm{c} / \mathrm{s}$, and there was delta activity which was bilaterally synchronous. Both of these frequencies were inhibited by visual stimuli. In Case 11, where the cerebellar defect was the result of a hyperpyrexial episode, the dominant rhythm was 8 to $9 \mathrm{c} / \mathrm{s}$, there was diffuse theta activity maximal in the frontal areas, and some scattered delta activity, which was inhibited to some extent by visual stimuli.

We have already alluded to the fact that in three cases clinical affection of the basal ganglia was detected. In these cases there were no great differences in the records from those in which the disorder was confined to the cerebellum, and it seems that here, as is also the case in typical Parkinsonism, there is no great change in electroencephalographic patterns as a result of the affection of the basal ganglia themselves.

\section{Correlation between Clinical Features and Electroencephalographic and Post-mortem Findings}

Post-mortem examination was carried out in Cases 7, 10, and 21. We feel, therefore, that more detailed analysis of these three cases is indicated.

Case 7.-G.B. was a man aged 50. In 1950 this patient, a keen and proficient darts player, first experienced difficulty in maintaining his high degree of accuracy at the game. Within two to three months he developed unsteadiness of the legs, and thereafter the coordination of the upper and lower limbs gradually deteriorated. In 1951, he experienced great difficulty with writing and began to have 'jumping' (myoclonic jerks) in both knees. The family history was not contributory. Examination on August 10, 1951, revealed that abnormalities, confined to the nervous system, comprised unsustained bilateral nystagmus, slight slurring dysarthria, generalized hypotonia, intermittent bilateral myoclonus of the hamstrings, dysdiadokokinesia, and impaired finger-nose and heelshin tests.

Analysis of the cerebrospinal fluid, radiographs of the skull and chest, and a Wassermann reaction and blood count all proved normal. No electroencephalogram was performed at that time.

During the next four years the condition steadily deteriorated and additional signs of a striatal type of tremor, with associated cog-wheel rigidity, supervened. He was re-admitted to hospital on July 1, 1955, when routine investigation again proved quite normal with the 
TABLE I

CLINICAL FINDINGS IN PRESENT SERIES GROUPED ACCORDING TO GREENFIELD (1954)

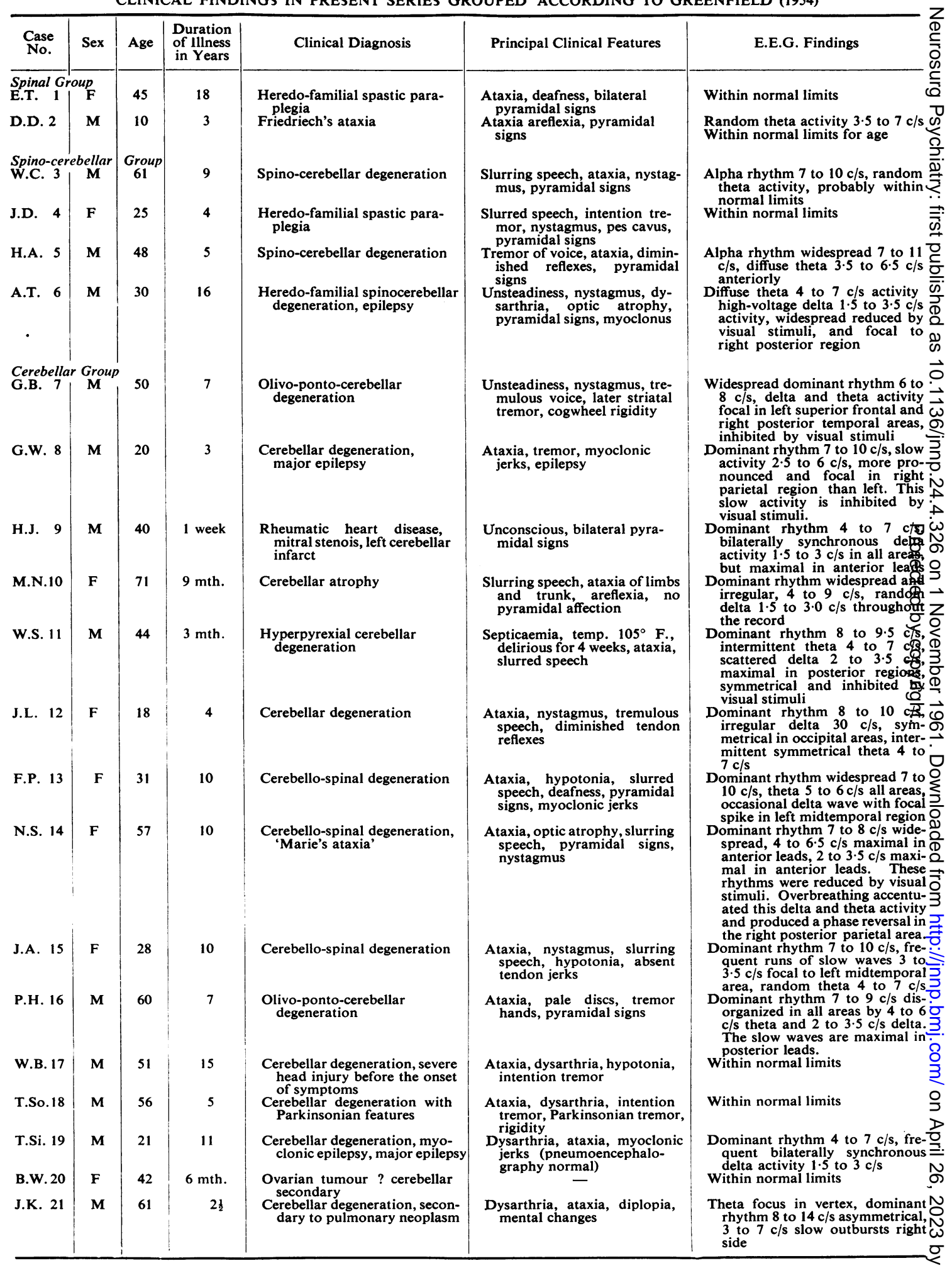


exception of the electroencephalogram. The dominant rhythm was slow (6 to $8 \mathrm{c} / \mathrm{s}$ ) and widespread. Medium voltage slow waves at $3.5 \mathrm{c} / \mathrm{s}$ occurred in all areas, frequently out of phase in the left frontal and left posterior temporal regions. His clinical condition continued to decline and he died on July 31,1955 . At no time was there any clinical evidence of affection of the pyramidal pathways, and his mental status was preserved throughout.

Dr. P. O. Yates, who carried out the necropsy, reported that the brain arteries were slightly atherosclerotic, the cerebellum was smaller than normal, the pons and inferior olives appeared atrophic.

The cerebellar folia showed in many places marked atrophic changes with loss of Purkinje cells and reduction of the granular layer. This change was scattered and does not show any regularity of pattern. Bergman's cells were increased in number and there were more gliotic nuclei in the molecular layer of the involved folia. The white matter showed a diffuse loss of myelin appropriate to the loss of neurones, with gliotic sclerosis. There were no particular changes in the structure of the dentate nuclei. The pons was very small and showed a moderate degree of gliosis, diffusely spread towards the medulla. There were a few foci of demyelination. The olivary nuclei did not show any pronounced changes, apart from a slight demyelination of nerve fibres. There was a slight diffuse gliosis throughout the medulla. The nuclei of both striothalamic groups showed increased satellitosis with slight gliosis. There was no change in the ganglion cells. Sections taken from the cerebrum showed no significant histological abnormality.

In short, a patient in whom there was widespread progressive affection of the cerebellar area with no obvious histological affection of the cerebrum, showed in life these definite changes in the electroencephalogram.

Case 10.-M.N. a woman, aged 71, in July, 1953, began to exhibit unsteadiness of gait which rapidly deteriorated so that within six months she was bedridden. She soon became ataxic in the trunk and upper limbs, and developed a slurring dysarthria. Examination on February 26, 1954 (Dr. G. F. Smyth), showed that the patient was quite uncooperative; she was extremely ataxic with irregular tremors of the hands at rest. The limbs were hypotonic. All reflexes were absent. Plantar responses were flexor. Investigations at that time revealed that abnormalities were again confined to the electroencephalogram. The record was dominated by a medium voltage moderately sustained irregular activity at 4 to $9 \mathrm{c} / \mathrm{s}$ fairly symmetrical and synchronous, but abnormally widespread. It was frequently displaced by medium to high-voltage delta waves at 1.5 to $3.5 \mathrm{c} / \mathrm{s}$ appearing at random throughout the record and often underlying the dominant rhythms. Occasionally a lowvoltage fast activity was noted. Her condition deteriorated rapidly and she died on April 10, 1954.

Dr. P. O. Yates carried out the necropsy and reported that a moderate degree of atheroma was seen in the coronary, carotid, and cerebral vessels. The cerebrum appeared normal. The cerebellar folia appeared shrunken throughout both hemispheres uniformly.

In various sections from the cerebrum there was no detectable abnormality. The cerebellum showed a thinning and loss of the granular layer. Purkinje cells appeared normal. Myelin was lost in the folia. The thalamus appeared normal, as did the spinal cord.

Once again, therefore, the widespread electroencephalographic changes appear to have been associated with degenerative changes in the cerebellum, producing quite marked clinical cerebellar dysfunction.

Case 21.-J.K., a man, aged 61, in 1946 developed a left-sided hemiparesis of sudden onset from which he made a full recovery within the space of three months. In February, 1954, he became constipated and began to lose weight. In May, 1954, he became unsteady and experienced difficulty in focusing. Shortly after this he became dysarthric and his gait rapidly more ataxic. His hands and arms also developed incoordination. Examination on July 27,1954 , showed sustained bilateral coarse nystagmus, dysarthria, ataxia of the arms, trunk, and legs, slight diminution of the left ankle jerk. Plantar responses were equivocal.

A barium meal and a barium enema were normal. A radiograph of the chest was normal but one of the pelvis showed Paget's disease. The serum alkaline phosphatase was 31.5 units $/ 100 \mathrm{ml}$. Cerebrospinal fluid was normal on resting pressure, and a cell count gave 14 lymphocytes, 1 mononuclear cell/per c.mm., protein $60 \mathrm{mg} . / 100 \mathrm{ml}$., globulin slight opalescence, Lange curve 1123210000 . An electroencephalogram on July 2 showed that the dominant rhythm was a well-sustained medium to high-voltage alpha activity at 8 to $9 \mathrm{c} / \mathrm{s}$. This activity was asymmetrical, spread well forward, and its higher voltage components were blocked by visual attention. The highest voltage slower alpha components, together with some medium to high voltage slow waves, at 3 to $7 \mathrm{c} / \mathrm{s}$ often appeared in short bursts and were out of phase in the central, parietal, and mid-temporal areas on the right side, one short burst at $3 \mathrm{c} / \mathrm{s}$ being of high voltage and focal in the centro-parietal leads. A repeat E.E.G. on September 17 showed a medium to high-voltage, well-sustained alpha rhythm at 8 to $11 \mathrm{c} / \mathrm{s}$ still asymmetrical, spreading well forward and blocked by visual attention. Medium voltage theta activity at 4 to $7 \mathrm{c} / \mathrm{s}$ was fairly prominent. Long runs of bilaterally synchronous medium voltage theta waves at 4 to $5 \mathrm{c} / \mathrm{s}$ appeared in the temporo-occipital leads, and were sometimes, but not always, more frequent on the right side. There were also infrequent bursts of a complex mixture of alpha, beta, theta, and delta waves out of phase in the right midtemporal area.

On clinical grounds a diagnosis of a subacute cerebellar degenerative process, probably secondary to an undisclosed neoplasm, was made. The patient was discharged and his subsequent course followed as an out-patient and later as an in-patient at the Withington Hospital, Manchester, where he was admitted under the care of Dr. Greenwood. The patient did not die until February $10,1956$. 
We are indebted to Dr. T. A. White for the postmortem report. 'The brain appeared superficially normal, and was fixed whole for further examination. Bronchopneumonia was noticed and, in addition, all the major bronchi were surrounded by a tumour which appeared to have originated near the region of the left upper main bronchus. The glands around the trachea bifurcation at the lung hilum were replaced by a solid rather nodular mass of white growth, which proved to be an oat-cell carcinoma. Sections from the cerebrum were normal, and from the cerebellum showed widespread loss of Purkinje cells with a granular ependymitis in the fourth ventricle. There was a little lymphocyte cuffing round some of the vessels. These findings are not inconsistent with Marie's degeneration.'

\section{Discussion}

As was indicated earlier, there has been some difficulty in explaining the abnormal wave patterns in posterior fossa tumours. Williams (1939) believed that oedema was responsible for the slow wave abnormalities and Scarff and Rahm (1941) showed that ventricular decompression resulted in rapid disappearance of the slow waves. Bagchi et al. (1952) stated that the background slowing and burst activity appeared most often in the cases with the most marked ventricular dilatation. Bickford and Baldes (1947) suggested that the bursts of slow waves were due to excessive diencephalitic discharges produced by an expanding third ventricle. The theory of direct occipital compression was emphasized by Ecker (1948).

The findings in our cases appear to indicate that slow waves and background slowing are likely to occur in cerebellar lesions quite independently of an increase in intracranial tension, and suggest that the cerebello-central connexions may be important in the production of electrical patterns. These findings are particularly interesting when viewed in relation to experimental evidence on the subject, for Walker (1938) showed that stimulation of the neocerebellar cortex of the cat produced high-voltage, of fast discharges in the opposite motor and premotor $\frac{3}{6}$ regions whilst Henneman, Cooke, and Snider (1948), 0 employing the technique of evoked potentials, were able to show quite definite and extensive cerebellocerebral relationships. It is not unreasonable to deduce, therefore, that a progressive defect of cerebellar structure will have its effect on the basic $\overrightarrow{\bar{c}}$ electrical patterns of the brain. An important corollary of these findings is that the identification of a posterior fossa lesion as space-occupying or degenerative in character must depend upon evidence other than electroencephalography, since the abnormal patterns displayed may occur in either type of lesion.

We are grateful to Dr. F. R. Ferguson and Dr. G. E. Smyth who gave us the opportunity of studying these patients under their care.

\section{REFERENCES}

Bagchi, B. K. Lam R. L. Kooi, K, A., and Bassett, R. C. (1952).

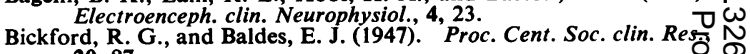
20, 87. Brain, W. R., and Strauss, E. B. (1955). Recent Advances in Neurolo宂 Daly, D., Whelan, J. L.. Bickford, R. G., and McCarthy, C. S. (1953

Dow, R. S. (1956). Ibid, 8, 165.

Ecker, A. (1948). J. Neurosurg. $5,51$.

Greenfield, J. G. (1954). The Spino-Cerebellar Degeneration Blackwell, Oxford. $P$. and Snider, R. S. (1948). Amer.

Henneman, E., Cooke, P., and Snider, R. S. (1948). Amer.
Physiol., 155, 443.

physiol., 1, 455 .
Scarff, J. E., and Rahm, W. E. Jr. (1941). J. Neurophysiol., 4, 418. Smyth, G. E. (1941). Brain, 64, 63.

Snider, R. S. (1950). Arch. Neurol. Psychiat. (Chicago), 64, 196. Snider, and Eldred, E. (1952). J. Neurophysiol., 15, 27.

Walker, A. E. (1938). Ibid, 1, 16.

Walter, W. G. (1936). Lancet, 2, 305.

(1937). Proc. roy. Soc. Med., 30, 33.

(1938). J. Neurol. Psychiat., 1, 359.

Williams, D. (1939). Brain, 62, 321. 